\title{
Molecular bases for the constitutive photomorphogenic phenotypes in Arabidopsis
}

Vinh Ngoc Pham, Xiaosa Xu' and Enamul Huq

Department of Molecular Biosciences and The Institute for Cellular and Molecular Biology, The University of Texas at Austin, Austin, TX 78712.

Corresponding author: Enamul Huq, University of Texas at Austin, NHB 2.616, Stop A5000, 100 East 24th St. Austin, TX 78712-1095. Tel: 512-471-9848, Fax: 512-4711218, e-mail: huq@austin.utexas.edu

Keywords: E3 Ubiquitin ligase, Phytochrome Interacting Factors, photomorphogenesis, 26S Proteasome degradation, Skotomorphogenesis, Arabidopsis.

Running title: Molecular bases for the cop phenotype in Arabidopsis

Summary statement: Strikingly similar morphological and gene expression phenotypes among copl, spaQ and pifQ mutants suggest that the cop phenotype of the copl and spaQ mutants might be due in part to a reduced level of PIFs.

present address: Cold Spring Harbor Laboratory, New York, NY 


\begin{abstract}
The transition from skotomorphogenesis to photomorphogenesis is regulated in part by COP1/SPA complex and PIFs in Arabidopsis. The constitutive photomorphogenic (cop) phenotypes of the copl and $\operatorname{spa} Q$ mutants were shown to be due to a high abundance of the positively acting transcription factors. Here we show that the four major PIF proteins are unstable in cop1 mutant, and an overexpression of PIF1, PIF3, PIF4 and PIF5 suppresses the copl phenotypes in the dark. A comparison of the transcriptome data among copl, spaQ and pifQ reveals remarkably overlapping gene expression profiles with a preferential regulation of the PIF direct target genes. Additionally, HFR1 strongly inhibits the in vivo binding and transcriptional activation activity of PIF1 in the dark. Taken together, these data suggest that the cop phenotypes of the copl and spaQ mutants might be due to a combination of the reduced level of PIFs, increased level of the positive factors (e.g., HY5/HFR1 and others), and the HFR1-mediated inhibition of PIF targeted gene expression in the dark.
\end{abstract}




\section{Introduction}

Plants are evolved with contrasting developmental programs for successful establishment of young postgerminating seedlings early in their life cycle. In darkness, plants undergo skotomorphogenesis defined by elongated hypocotyls, an apical hook and closed cotyledons. This developmental program is suited for protection of the apical region during rapid emergence of the seedlings through the soil surface. Once the seedlings are exposed to ambient light, they undergo photomorphogenesis defined by short hypocotyls, an absence of apical hook, open, expanded and green cotyledons. This growth pattern allows seedling body plan formation for maximal light capture and autotrophic growth (Gommers and Monte, 2018). Photomorphogenesis has been proposed to be the default pathway for plant development as a series of mutants displaying constitutive photomorphogenic (cop) phenotypes in the dark has been described (Xu, et al, 2015). These include 11 loci encoding the CONSTITUTIVE PHOTOMORPHOGENIC1/DE-ETIOLATEDI/FUSCA (COP/DET/FUS) genes (Lau and Deng, 2012), four loci encoding SUPPRESSOR OF PHYA-105 (SPA1-SPA4) (Laubinger, et al, 2004) and a small family of basic helix loop helix (bHLH) transcription factor genes called the PHYTOCHROME INTERACTING FACTORs (PIF1-PIF8) (Leivar and Quail, 2011; Pham, et al, 2018b). These genes encode repressor proteins that act additively and/or synergistically to prevent precocious germination and seedling establishment in the dark.

Among these repressors, COP1 functions as an E3 ubiquitin ligase in association with SPA1-SPA4 targeting a variety of substrates including the positively acting transcription factors (e.g., HY5/HFR1/LAF1 and others) in light signaling pathways for Ubiquitin/26S proteasome-mediated degradation (Hardtke, et al, 2000; Hoecker, 2017; Jang, et al, 2005; Osterlund, et al, 2000; Seo, et al, 2003; Yang, et al, 2005b). COP1, SPAs and CUL4 form CUL4 ${ }^{\text {copr.sps }}$ E3 ubiquitin ligase complexes that target positively acting factors in the dark (Zhu, et al, 2008). Consistently, copl, spaQ and cul4cs (cosuppressed) lines display constitutive photomorphogenic (cop) phenotypes in the dark. In addition, another complex called the COP9 signalosome (CSN) consists of eight distinct subunits (CSN1-CSN8), which is involved in deconjugation of the NEDD8/RUB1 to the 
CULLIN RING ligases (CRLs) (Lau and Deng, 2012; Serino and Deng, 2003). Mutation in any of these subunits also display cop phenotypes in the dark.

DET1 is a nuclear protein, which binds to the $\mathrm{N}$-terminal tail of histone $\mathrm{H} 2 \mathrm{~B}$ and regulates cell-type-specific expression of light-regulated genes (Benvenuto, et al, 2002; Pepper, et al, 1994). DET1 also promotes skotomorphogenesis in part by stabilizing PIFs in the dark (Dong, et al, 2014). In addition, DET1 suppresses seed germination by destabilizing HFR1 and stabilizing PIF1 (Shi, et al, 2015). DET1 also interacts with COP10 and DAMAGED DNA BINDING PROTEIN 1 (DDB1) to form CUL4 ${ }^{\mathrm{cD}}$ complex that represses photomorphogenesis in the dark in part by degrading positively acting transcription factors (Chen, et al, 2006; Schroeder, et al, 2002).

PIFs belong to the basic helix-loop-helix (bHLH) family of transcription factors that repress photomorphogenesis in the dark by promoting skotomorphogenic development. There are eight PIFs (PIF1-PIF8) in Arabidopsis with a high degree of sequence similarity (Pham, Kathare and Huq, 2018b). However, individual pif mutants display distinct phenotypes that are especially pronounced in the four major pif mutants (pif1, pif3, pif4 and pif5 collectively called PIF quartet). For example, pif1 seeds germinate under red and far-red light as well as in darkness (Oh, et al, 2004; Shen, et al, 2005), suggesting PIF1 is a repressor of light-induced seed germination. Both pifl and pif3 mutants have more chlorophyll and carotenoids compared to wild type during the transition from dark to light (Huq, et al, 2004; Moon, et al, 2008; Stephenson, et al, 2009; Toledo-Ortíz, et al, 2010), suggesting that PIF1 and PIF3 suppress the biosynthesis of these pigments. pif3, pif4 and pif5 mutants display hypersensitive phenotypes in response to red light, in part by inducing co-degradation of these PIFs with phyB (Huq and Quail, 2002; Khanna, et al, 2007; Monte, et al, 2004; Zhu and Huq, 2014). In this process, multiple kinases (e.g., PPKs) and E3 Ubiquitin Ligases (e.g., CUL3 ${ }^{\text {гвв }}$ ) participate in inducing the co-degradation of PIFs and phyB in response to light (Ni, et al, 2017; Ni, et al, 2014; Pham, Kathare and Huq, 2018b). Thus, phyB is more abundant in these mutants resulting in hypersensitive phenotypes under red light. In addition, other kinases (e.g., PPKs. BIN2 and CK2) and E3 Ubiquitin Ligases (e.g., CUL1 ${ }^{\text {ввг/2 }}$, CUL3 ${ }^{\text {вор }}$ and CUL4 ${ }^{\text {copr.spA }}$ ) induce the degradation of PIFs in response to light in a phytochrome-dependent manner to promote photomorphogenesis (Bernardo-García, et al, 2014; Bu, et al, 2011; Dong, et 
al, 2017; Ni, Xu, González-Grandío, et al, 2017; Pham, Kathare and Huq, 2018b; Zhang, et al, 2017). Strikingly, the quadruple mutant of the PIF quartet, pifQ (pif1 pif3 pif4 pif5) displays constitutive photomorphogenesis in the dark (Leivar, et al, 2008; Shin, et al, 2009), suggesting that these PIFs repress photomorphogenesis in the dark. They do so by regulating gene expression directly and indirectly in an individual to a shared manner (Pfeiffer, et al, 2014; Pham, Kathare and Huq, 2018b).

The cop phenotypes of the copl and spaQ mutants were thought to be primarily due to a high abundance of the positively acting transcription factors (e.g., HY5/HFR1/LAF1 and others) in the dark (Hoecker, 2017). However, a few reports showed that PIFs are less abundant in copl (Bauer, et al, 2004; Shen, et al, 2008; Xu, et al, 2017; Zhu, et al, 2015) and also to a lesser extent in the spaQ mutants (Leivar, Monte, Oka, et al, 2008; Ni, Xu, Tepperman, et al, 2014), suggesting that the instability of PIFs might contribute to the cop phenotypes of these mutants. Here we show that the gene expression signature of copl and $\operatorname{spa} Q$ overlaps with pifQ in the dark with a preferential targeting of PIF direct target genes, suggesting that the cop phenotype of the copl and spaQ is partly due to a reduced level of PIFs in these backgrounds. In addition, we also show that the positively acting transcription factor HFR1 strongly suppresses PIF1 function by sequestration; thereby, promoting the cop phenotypes of the copl and spaQ in the dark.

\section{Results}

\section{COP1 and SPA positively regulate PIF protein level in darkness}

The constitutive photomorphogenic (cop) phenotypes of the cop1-4, spaQ and pifQ have been previously described (Figure 1A) (Deng, et al, 1992; Laubinger, Fittinghoff and Hoecker, 2004; Leivar, Monte, Oka, et al, 2008; Shin, Kim, Kang, et al, 2009). To examine if the cop phenotype of the pifQ is due to a reduction in the COP1 level, we performed immunoblot using an anti-COP1 antibody for four-day-old darkgrown seedlings of wild type, cop1-4, spaQ and pifQ. Results show that the COP1 level in pifQ and $\operatorname{spaQ}$ is similar to that in the wild type seedlings (Figure 1B), suggesting that the pifQ phenotype is not due to a reduction in the COP1 level. 
Previously, we and others have shown that the PIF levels in the copl and spaQ mutants are reduced compared to wild type in the dark (Bauer, Viczian, Kircher, et al, 2004; Leivar, Monte, Oka, et al, 2008; Zhu, Bu, Xu, et al, 2015). To systematically analyze PIF levels without the transcriptional regulation in these mutants, we crossed the overexpression lines of tagged PIF 1, PIF3, PIF4 and PIF5 using the constitutively active $35 \mathrm{~S}$ promoter in the cop1-4, and TAP-PIF1 and PIF5-Myc in spaQ mutant backgrounds, and performed immunoblots for protein levels. Results show that all four PIF levels are reduced in the copl-4 as previously shown (Figure 1C-F). Both TAP-PIF1 and PIF5-Myc levels are reduced in the spaQ compared to wild type (Figure S1). Thus, the cop phenotype of the copl-4 and spaQ might be in part due to a reduction in the PIF levels in these backgrounds.

We also examined the transcript levels of the native PIFs in the copl-4 and spaQ mutants using quantitative RT-PCR assays (Figure S2). The transcript levels of PIF1, PIF3 and PIF4 are similar between Col-0 and cop1-4, except the transcript level of PIF5 is strongly increased in copl-4. In spaQ mutant, the transcript levels of PIF1, PIF3 and PIF4 were slightly lower, while PIF5 transcript is slightly higher compared to wild type seedlings. These data illustrate that COP1 and SPA proteins positively regulate PIF protein levels in darkness possibly by destabilizing HFR1, as HFR1 has been shown to induce degradation of PIF1 by heterodimerization (Xu, Kathare, Pham, et al, 2017).

\section{PIFs are degraded in the copl-4 and spaQ mutants through the 26S proteasome}

PIFs are known to be stable in the dark and undergo degradation in light through the 26S proteasome pathway (Pham, Kathare and Huq, 2018b). However, a recent study showed that PIF1 is also degraded in the dark by direct heterodimerization with HFR1 (Xu, Kathare, Pham, et al, 2017). To test whether the degradation of other PIFs in the copl-4 and spaQ backgrounds in the dark is also through the $26 \mathrm{~S}$ proteasome pathway, we treated the dark-grown seedlings with the proteasome inhibitor (Bortezomib) for 4 hours and then extracted total protein for immunoblots. Results show that the proteasome inhibitor prevents the degradation of all four PIFs in the cop1-4 background (Figure 2). Both TAP-PIF1 and PIF5-Myc are also stabilized in the spaQ background upon 
Bortezomib treatment (Figure S1). These data suggest that COP1/SPA complex stabilizes PIFs in the dark presumably by destabilizing HFR1.

\section{Overexpression $P I F$ s partially suppresses the cop phenotypes of cop1-4 and spaQ}

If the reduced level of PIFs in the copl-4 and spaQ backgrounds contributes to the cop phenotypes of these mutants, we hypothesized that an overexpression of these PIFs in the copl and spaQ mutants is expected to suppress the cop phenotypes. To test this hypothesis, we overexpressed four PIFs (TAP-PIF1, PIF3-Myc, PIF4-Myc and PIF5Myc) in the copl-4 and two PIFs (TAP-PIF1 and PIF5-Myc) in the $s p a Q$ backgrounds and examined their phenotypes in the dark. Results show that while the hypocotyl lengths of TAP-PIF1/cop1-4 and PIF3-Myc/cop1-4 are comparable to those of cop1-4, the hypocotyl lengths of PIF4-Myc/copl-4 and PIF5-Myc/copl-4 are significantly longer compared with copl-4 (Figure 3A-D). Moreover, all four PIF overexpression lines in the cop 1-4 mutant displayed significantly smaller cotyledon angle compared to that of cop14, suggesting that PIFs suppress the cop phenotypes of cop1-4. These data also suggest that the cop phenotype of copl-4 and $\operatorname{spaQ}$ are partially due to a reduced level of PIFs.

\section{cop1-4, spaQ and pifQ display a large overlapping set of co-regulated genes}

Previously, the cop phenotypes of the copl mutant was demonstrated to be mainly due to the high abundance of the positive regulators (e.g., HY5/HFR1/LAF1 and others) in the dark (Hoecker, 2017). Because PIFs are unstable in the copl and spaQ mutants in the dark (Figure 1) (Bauer, Viczian, Kircher, et al, 2004; Leivar, Monte, Oka, et al, 2008; Zhu, Bu, Xu, et al, 2015), we hypothesized that COP1-, SPA- and PIF-regulated genes might overlap in the genome wide expression analyses. To test this hypothesis, we analyzed the results from a recent RNA-Seq experiment using the cop1-4, spaQ and pifQ mutant seedlings grown in darkness (Pham, et al, 2018a; Zhang, et al, 2013). Although these experiments were performed in two different laboratories and growth conditions were slightly different, the results show that a large proportion of the differentially expressed genes (1120) overlap among cop1-4, spaQ and pifQ (Figure 4A, B; Dataset S1). Approximately, 39\% percent of the PIF-regulated genes display overlapping expression patterns with the COP1- and SPA-regulated genes. Among these 1120 genes, 483 genes are up-regulated and 431 genes are down-regulated in all three backgrounds 
compared to wild type (Figure 5A, B). Interestingly, 206 of the PIF-regulated genes display opposite regulation from the COP1- and SPA-regulated genes (Figure 4A, B; Dataset S3).

To identify the biological processes controlled by these co-regulated genes, we performed Gene Ontology (GO) analyses of the co-regulated genes and divided into two classes: up-regulated and down-regulated genes (Figure 4C, D; Dataset S2). A total of 94 enriched GO terms have been identified for these co-regulated genes. The co-upregulated genes are enriched in chlorophyll biosynthetic process, defense responses, photosynthesis, response to light stimulus (including red light and blue light), response to cold and cytokinin. The co-downregulated genes are involved in regulation of transcription, cell wall organization, response to hormones (abscisic acid and auxin), response to red light and also metabolic processes. These results are consistent with the cop phenotypes of these mutants.

Interestingly, 206 of the PIF-regulated genes display opposite regulation from the COP1- and SPA-regulated genes (Figure 4A, B; Dataset S3). GO analyses of the oppositely regulated genes (206 genes) between pifQ and copl-4/spaQ using Database for Annotation, Visualization and Integrated Discovery (DAVID) (Dataset S3) and GO Analysis Toolkit and Database for Agricultural Community (AgriGo) (Figure S4 and S5) show that PIFs function oppositely compared to COP1 and SPA proteins in a few biological processes. For example, many genes involved in responses to UV-B and flavonoid biosynthesis are down-regulated in pifQ, while they are up-regulated in copl-4 and $\operatorname{spaQ}$ (Figure S4; Dataset S3). Similarly, many other genes involved in defense responses, salicyclic acid (SA) metabolism and signaling are up-regulated in pifQ, while they are down-regulated in copl-4 and spaQ (Figure S5; Dataset S3. Thus, while PIFs and COP/SPA complex repress photomorphogenesis coordinately, they also function antagonistically in a few biological processes.

\section{Direct target genes of PIFs are co-regulated in cop1-4 and spaQ}

If COP1 and SPA repress photomorphogenesis in the dark in part by stabilizing PIFs, then the PIF direct target gene expression is expected to be affected in copl-4 and spaQ. Interestingly, among 338 PIF direct target genes (Pfeiffer, Shi, Tepperman, et al, 
2014), 170 (>50\%) genes are co-regulated by copl-4 and spaQ (Figure 5A; Dataset S4). Furthermore, among the 209 PIF-induced genes, 110 genes are down-regulated in copl-4 and spaQ. In addition, among 129 PIF-repressed genes, 42 genes are up-regulated in cop1-4 and spaQ backgrounds (Figure 5A; Dataset S4). GO analyses revealed that the majority of these genes function in responses to red, far-red light signaling, auxin responses and regulation of transcription. Strikingly, the degree and direction of expression of these PIF direct genes are very similar among all three mutant groups.

To verify the RNAseq data by an independent method, we selected a subset of PIF direct target genes involved in auxin responses, cell wall organization and photosynthesis, and performed RT-qPCR analyses to determine the relative expression patterns in the copl-4, spaQ and $p i f Q$ in comparison to wild-type. Results show a strikingly similar pattern among cop1-4, spaQ and pifQ for both PIF-induced and repressed genes (Figure 5C, D), consistent with the RNAseq data (Figure 5D, E). These data suggest that the cop phenotype of the copl and $\operatorname{spa} Q$ mutants might be partly due to the reduced level of PIFs and their target gene expression.

\section{HFR1 represses the transcriptional activity of PIF1}

Previously, it was shown that HFR1, a HLH transcription factor is more abundant in the copl-4 and spaQ backgrounds compared to wild type (Hoecker, 2017). Because HFR1 inhibits the DNA binding activity of PIFs (Shi, et al, 2013), and the PIF levels are reduced in the copl-4 and spaQ (Figure $1 \mathrm{~B}-\mathrm{F}$; Figure S1A), we hypothesized that the high abundance of HFR1 in the copl-4 and spaQ backgrounds might contribute to the cop phenotypes of these mutants. To test this hypothesis, we selected a subset of the PIF1 direct target genes that are also known to be regulated by HFR1 and performed the RTqPCR analyses from dark-grown seedlings of wild type Col-0, pifQ, cop1-4 and cop1-4 $h f r 1$. Results show that the selected genes are expressed at a reduced level in both pifQ and copl-4 mutant backgrounds similar to the RNAseq data (Figure 6A). Strikingly, the expression level of these genes is much higher in the copl-4 hfrl double mutant background compared to cop 1-4 (Figure 6A). However, the increased expression of the PIF1 target genes in the copl-4 hfrl might be either due to the high PIF1 protein level, and/or due to the loss of HFR1's suppression of PIF1's DNA binding activity. To 
distinguish between these possibilities, we performed the chromatin immunoprecipitation followed by quantitative PCR (ChIP-qPCR) for dark-grown seedlings expressing the TAP-PIF1 fusion protein in the copl-4 and copl-4 hfrl backgrounds. We also examined the immunoprecipitated TAP-PIF1 protein level in all these backgrounds during the ChIP assay (Figure 6B, inset), and divided the promoter enrichments by the protein levels for each genotype to calculate the relative promoter occupancy of PIF1 independent of PIF1 protein level. Results show that the immunoprecipitated TAP-PIF1 protein level is lower in the copl-4 background, but higher in the copl-4 hfrl double mutant background compared to the TAP-PIF1 only as previously reported (Figure 6B, inset) (Zhu, Bu, Xu, et $a l, 2015)$. The relative promoter occupancy of PIF1 show that the DNA binding activity of TAP-PIF1 was also reduced in the copl-4 background and increased in the copl-4 hfrl background (Figure 6B). These data further extend the recent report that HFR1 suppresses PIF1's DNA binding activity not only in imbibed seeds (Shi, Zhong, Mo, et al, 2013), but also in seedlings. Thus, HFR1 not only regulates the protein abundance but also the DNA binding activity of PIF1 in etiolated seedlings.

To examine the significance of regulation of PIF1 by HFR1, we measured the hypocotyl lengths for the dark-grown seedlings of wild-type, copl-4, copl-4 hfrl, copl4/TAP-PIF1, cop1-4 hfrl/TAP-PIF1 and TAP-PIF1. Results show that the hypocotyl lengths of the copl-4 hfrl/TAP-PIFl are much longer than that of the copl-4 hfrl backgrounds (Figures 6C, D). The hypocotyl length of the cop 1-4 is similar to that of the cop1-4/TAP-PIF1 possibly due to the reduced level of TAP-PIF1 protein level and/or increased sequestration of TAP-PIF1 by HFR1 in the cop1-4 background. Thus, TAPPIF1 has an increased function in regulating hypocotyl lengths in the copl-4 hfrl background compared to only copl-4.

\section{Discussion}

Analyses of the cop mutants have played an important role in our understanding of the light signaling pathways in plants. The prevailing view of the molecular basis of the cop phenotype is that the increased abundance of the positively acting transcription factors (e.g., HY5/HFR1/LAF1 and others) in the cop1-4 and spaQ mutants in the dark results in the cop phenotypes under darkness (Jang, Yang, Seo, et al, 2005; Osterlund, 
Hardtke, Wei, et al, 2000; Saijo, et al, 2003; Seo, Yang, Ishikawa, et al, 2003; Yang, et al, 2005a; Yang, Lin, Sullivan, et al, 2005b). Although several studies reported a reduced abundance of PIFs in various cop mutants compared to wild type (Bauer, Viczian, Kircher, et al, 2004; Leivar, Monte, Oka, et al, 2008; Ni, Xu, Tepperman, et al, 2014; Shen, Ling, Castillon, et al, 2008; Zhu, Bu, Xu, et al, 2015), the mechanism of this reduction and its contribution to the cop phenotype remain unknown. Here we show biochemical, molecular and genomic evidence supporting the hypothesis that the reduced PIF levels in the copl and spaQ mutants contribute to their cop phenotypes. First, we show that PIFs are actively degraded in the dark in the copl-4 and spaQ backgrounds through the $26 \mathrm{~S}$ proteasome pathway (Figures 1, S1). Second, the genome wide gene expression patterns largely overlap between COP1-, SPAs- and PIF-regulated genes with an altered expression of a set of PIF direct target genes (Figures 4, 5). Third, PIF1 is sequestered in the copl-4 background by increased abundance of HFR1 and possibly other HLH proteins, resulting in the reduced PIF activity in the cop1-4 background (Figure 6). Fourth, an overexpression of PIF1 in the copl-4 hfrl background promotes hypocotyl elongation in the dark. Fifth, an overexpression of four major PIFs in the copl4 background suppresses the cop phenotypes of the copl-4 (Figure 3). Overall, these data strongly suggest that the reduction in the PIF levels and PIF activity in the copl-4 and spaQ backgrounds contribute to their cop phenotypes.

Despite similar morphological and molecular phenotypes among cop1-4, spaQ and $p i f Q$, the GO analyses of the differentially expressed genes oppositely regulated between pifQ and cop1-4/spaQ reveals that these mutants also have distinct roles in plant signaling pathways. One of the striking differences is in the enrichment of the genes involved in the SA metabolism and signaling in pifQ compared to cop1-4 and spaQ, suggesting that PIFs might suppress defense responses as previously discussed (Paik, et $a l, 2017)$. In fact, PIFs are known to promote growth possibly by suppressing the defense responses, as a trade-off between growth vs defense is a well-known phenomenon in plant growth and development (Paik, Kathare, Kim, et al, 2017). In contrast, the genes involved in the UV-B responses and flavonoid biosynthesis are down-regulated in pifQ, while they are upregulated in cop1-4 and spaQ. Although, a role for PIFs in the UV-B signaling has not been examined in detail yet, a recent study implicated that PIFs might 
be involved in UV-B-induced leaf hyponasty (Fierro, et al, 2015). However, COP1 and SPA proteins function positively in the UV-B signaling pathways (Huang, et al, 2013; Tilbrook, et al, 2013). Overall, these analyses highlight both common and distinct functions of PIFs, COP1 and SPA proteins in regulating biological processes in plants.

One of the sources of the opposite functions between PIFs and COP1/SPA complex is the increased abundance of the positively acting transcription factors especially HFR1 in the cop1-4 and spaQ backgrounds. HFR1 is an atypical bHLH protein that sequesters PIF activity as well as reduces the PIF abundance (Figure 6) (Hornitschek, et al, 2009; Shi, Zhong, Mo, et al, 2013; Xu, Kathare, Pham, et al, 2017). Similar to HFR1, HECATE family of bHLH proteins also inhibits PIF activity, and is degraded in the dark possibly by COP1/SPA complex (Zhu, et al, 2016). Thus, COP1/SPA complex might negatively regulate the abundance of factors that function antagonistically to PIFs.

In summary, we propose a revise model for the molecular bases of the cop phenotypes in plants (Figure 7). First, as previously hypothesized, an increased abundance of the positively acting transcription factors (e.g., HY5, LAF1, HFR1 and others) in the copl and spaQ mutants promotes photomorphogenesis in the dark. Second, a reduced level of PIFs in the copl and spaQ mutants contributes to the cop phenotype in the dark. Finally, a reduction in PIF activity due to the increased abundance of the atypical bHLH proteins (e.g., HFR1, HECATE and possibly others) in copl and spaQ mutants additively promotes the cop phenotypes. It is notable that all three activities are tightly linked to each other contributing in concert to the skotomorphogenic and photomorphogenic developments in plants.

\section{Materials and Methods}

\section{Plant materials, growth conditions and measurements}

Columbia-0 (Col-0) ecotype of Arabidopsis thaliana seeds were used for all experiments. Seeds were surface sterilized and then plated on the Murashige and Skoog (MS) medium without sucrose. After stratified at $4^{\circ} \mathrm{C}$ for 3 days, seeds were exposed to white light for 3 hours at room temperature to trigger the seed germination before placing them back in the dark for an additional 4 days. These 4-day-old seedlings were used for protein extraction for Western blots, measurements of hypocotyl lengths and cotyledon 
opening angle phenotypes. A total of 90 seedlings from three biological replicates were measured for hypocotyl lengths and cotyledon opening angles using Image J software (http://rsb.info.nih.gov/ij/). Significant difference between different genotypes was determined using one-way ANOVA and Tukey's HSD tests, indicated by different letters in the figures.

\section{Generation of transgenic lines}

The pif1, pifQ, cop1-4, cop1-4pif1, cop1-4/TAP-PIF1 (Castillon, et al, 2009; Xu, et al, 2014; Zhu, Bu, Xu, et al, 2015), TAP-PIF1 (Bu, Zhu, Yu, et al, 2011), PIF3-Myc (Park, et al, 2004), PIF4-Myc (Shor, et al, 2017) and PIF5-Myc (Sakuraba, et al, 2014) plants were previously published. PIF overexpression lines were crossed with cop 1-4 and spaQ mutants. The crossed homozygous lines were selected from F3 population using antibiotic selection. The copl-4 mutant was selected by sequencing. The spaQ homozygous lines were selected by genotyping spa mutants. Primers used for sequencing and genotyping are listed in Table S1. For generation of copl-4 hfrl/TAP-PIF1, cop-4 hfrl was crossed into TAP-PIF1 to obtain F1 generation. Through genotyping and antibiotic selection (Gentamycin) of the F2 and F3 generation, we obtained copl-4 hfr 1/TAP-PIF1. Primers used for sequencing and genotyping are listed in Table S1.

\section{Transcriptomic analyses}

To evaluate and compare the differential gene expression in copl-4 and spaQ, the raw read counts were obtained from our previous RNA-seq study (Pham, Hoecker and Huq, 2018a). Raw data and processed data for the total read counts of sequencing reads in Col-0, copl-4 and spaQ can be accessed from Gene Expression Omnibus database under accession number GSE112662.

RNA-seq was performed using 3-day-old dark-grown seedlings. Seeds were kept in the dark for 3 days at $4{ }^{\circ} \mathrm{C}$ and exposed to $3 \mathrm{hrs}$ of while light. After $21 \mathrm{~h}$ in the dark, plates were then treated with $2000 \mu_{\text {molm }}{ }^{2}$ far-red light for the true-dark condition as previously described (Leivar, Monte, Oka, et al, 2008). Total RNA was extracted after 2 days in the dark. 
For the RNA-seq analysis, raw read quality was accessed using FastQC (http://www.bioinformatics.babraham.ac.uk/projects/fastqc/). Raw reads were then aligned to the Arabidopsis genome using Bowtie2 (Langmead and Salzberg, 2012) and TopHat (Trapnell, et al, 2012). The annotation of the Arabidopsis genome was obtained from TAIR10 (https://www.arabidopsis.org/). Read count data were performed by HTseq (Anders, et al, 2015) (http://htseq.readthedocs.io/en/master/index.html).

Differentially expressed genes in copl-4/WT and spaQ/WT were identified using DESeq2 package (Love, et al, 2014). The differential gene expression was defined as those differ by $\geq 2$-fold with adjusted $P$ value $($ FDR $) \leq 0.05$.

Differentially expressed genes in pifQ and PIF differential direct-target genes list were obtained from RNA-seq and ChIP-Seq data, respectively, under the accession number GSE43286 (Pfeiffer, Shi, Tepperman, et al, 2014). Venn diagrams were generated using Venny 2.1.0 (http://bioinfogp.cnb.csic.es/tools/venny/). Heatmaps were generated using DESeq2 and ComplexHeatmap package $(\mathrm{Gu}$, et al, 2016) in the $\mathrm{R}$ statistical program. Gene Ontology (GO) enrichment analyses were performed using Database for Annotation, Visualization and Integrated Discovery (DAVID) v6.8 https://david.ncifcrf.gov/. GO bar graphs were generated based on the significant enriched terms with the lowest $\mathrm{p}$ value and FDR $(\leq 0.05)$ for GO terms. Hieratical graph results for the GO term analysis of copl-4, spaQ and pifQ regulated genes were also performed by GO Analysis Toolkit and Database for Agricultural Community (AgriGo http://bioinfo.cau.edu.cn/agriGO/index.php).

\section{Quantitative RT-PCR assay}

For determining the transcript levels of PIFs and PIF-direct target genes by RTqPCR assays, total RNA was extracted from the seedlings grown under the same conditions used for the RNA-seq experiment. One $\mu \mathrm{g}$ of total RNA treated with oncolumn DNase I (Sigma Aldrich, St. Louis, MO) was reversed transcribed using M-MLV reverse transcriptase (Thermo Fisher Scientific, Bartlesville, OK). RT-qPCR assay was performed using Power SYBR ${ }^{\circledR}$ green (Applied Biosystems, Foster City, CA). Gene specific primers are listed in Table S1. PP2A (At1g13320) was used as the internal 
control to normalize the expression of different genes. The calculation of the levels of expression of different gene relative to $P P 2 A$ is as follows: $2^{\star a c}$, where $\Delta \mathrm{Ct}=\mathrm{Ct}(P P 2 A)-$ $\mathrm{Ct}$ (specific gene) and $\mathrm{Ct}$ indicates the cycle threshold values. Relative expression was quantified from three biological replicates. Error bars indicate mean \pm SD. Student's t-test assuming unequal variances was performed, $P$ values are indicated in each figure.

\section{Protein extraction and immunoblot analyses}

For examination of COP1 protein level, $0.2 \mathrm{~g}$ tissue from 4-day-old dark-grown seedlings was extracted in extraction buffer as described ( $\mathrm{Zhu}, \mathrm{Bu}, \mathrm{Xu}$, et al, 2015). Total protein was separated on $8 \%$ SDS-polyacrylamide gel electrophoresis (PAGE). Proteins were transferred to PVDF membrane and Western blots were detected with anti-COP1 or anti-RPT5 (Enzo Life Sciences, Farmingdale, NY) antibodies for endogenous COP1 and RPT5, respectively.

For PIF protein levels in Col-0 and mutant backgrounds, total protein from 50 seedlings was extracted using $50 \mu \mathrm{l}$ urea extraction buffer $(48 \%$ urea $(\mathrm{w} / \mathrm{v}), 0.1 \mathrm{M}$ phosphate buffer $\mathrm{pH} 6.8,10 \mathrm{mM}$ Tris- $\mathrm{Cl} \mathrm{pH} 6.8,1 \mathrm{mM}$ phenylmethylsulfonyl fluoride (PMSF) and $1 \times$ protease inhibitor cocktail). Samples were centrifuged at 16,000g for 10 min and heated $65^{\circ} \mathrm{C}$ for $10 \mathrm{~min}$. Supernatants were analyzed on 6.5\% SDS- PAGE gels and detected using anti-Myc (dilution 1/1000, Catalog number OP10-200UG, EMD Millipore, MA) and anti-RPT5 antibodies (dilution 1/3000, Catalog number: BMLPW8245-0100, Enzo Life Sciences, Farmingdale, NY).

For treatment of $26 \mathrm{~S}$ proteasome inhibitor (Bortezomib, catalog number B-1408, LC Laboratories), 4-day-old dark-grown seedlings were transferred to $5 \mathrm{~mL}$ liquid $\mathrm{MS}$ media containing $40 \mu \mathrm{M}$ Bortezomib and incubated in the dark for 4 hrs. Total protein was then extracted using the urea extraction buffer as described above. For quantitation of protein levels, ImageJ software was used to measure the band intensities from three independent biological replicates, and normalized to RPT5 protein levels. Error bars indicate mean $\pm \mathrm{SD}$. Student's t-test assuming unequal variances was performed, $P$ values are indicated in each figure. 


\section{Chromatin immunoprecipitation (ChIP) assay}

The ChIP-qPCR assays were performed on 3-day-old dark-grown seedlings expressing TAP-PIF1 fusion protein in copl-4 and copl-4hfrl backgrounds. Anti-Myc (rabbit) antibody was used to immunoprecipitate TAP-PIF1 and associated DNA. DNA was amplified using primers specific to the G-box fragment or control regions. Anti-Myc (mouse) antibody was used to determine the immunoprecipitated TAP-PIF1 protein level in each background. Both the TAP-PIF1 promoter enrichment from the ChIP-qPCR and TAP-PIF1 protein level quantified by ImageJ were set as 1 . The relative enrichment of the fold-change of the cop1-4/TAP-PIF1 and cop1-4hfr1/TAP-PIF1 were first normalized compared with the TAP-PIF1 only for their promoter enrichment and protein levels, respectively, and then divided the promoter enrichments by the protein levels for each repeat. Final averages of three independent biological repeats for each genotype were calculated and shown as the bar graph. One biological repeat of the TAP-PIF1 protein level was shown as an example in inset. Error bars indicate standard deviation $(n=3$ independent biological repeats).

\section{Acknowledgments}

We thank Drs. Xing Wang Deng for sharing copl mutant, Ute Hoecker for sharing spaQ mutant, Giltsu Choi for sharing PIF3-Myc and PIF5-Myc seeds and Woe Yeon Kim for COP1 antibody. We thank the Huq lab members for the technical support and critical reading of the manuscript.

\section{Competing interests}

The authors declare no competing financial interests.

\section{Author contributions}

V.N.P., X.X. and E.H. designed experiments. V.N.P., and X.X. carried out experiments. V.N.P., X.X. and E.H. analyzed data and interpreted the results. V.N.P. wrote the article. V.N.P., X.X. and E.H. edited the manuscript. 


\section{Funding}

This work was supported by grants from the National Institute of Health (NIH)

(GM-114297) and National Science Foundation (MCB-1543813) to E.H.

\section{Supplementary material}

Supplementary material is available online. 


\section{References:}

Anders, S., Pyl, P. T. and Huber, W. (2015) HTSeq - a Python framework to work with high-throughput sequencing data. Bioinformatics, 31, 166-169.

Bauer, D., Viczian, A., Kircher, S., Nobis, T., Nitschke, R., Kunkel, T., Panigrahi, K. C., Adam, E., Fejes, E., Schafer, E., et al (2004) Constitutive photomorphogenesis 1 and multiple photoreceptors control degradation of phytochrome interacting factor 3 , a transcription factor required for light signaling in Arabidopsis. Plant Cell, 16, 1433-1445.

Benvenuto, G., Formiggini, F., Laflamme, P., Malakhov, M. and Bowler, C. (2002) The Photomorphogenesis Regulator DET1 Binds the Amino-Terminal Tail of Histone H2B in a Nucleosome Context. Current Biology, 12, 1529-1534.

Bernardo-García, S., de Lucas, M., Martínez, C., Espinosa-Ruiz, A., Daviere, J.-M. and Prat, S. (2014) BR-dependent phosphorylation modulates PIF4 transcriptional activity and shapes diurnal hypocotyl growth. Genes \& Development, 28, 1681-1694

Bu, Q., Zhu, L., Yu, L., Dennis, M., Lu, X., Person, M., Tobin, E., Browning, K. and Huq, E. (2011) Phosphorylation by CK2 enhances the rapid light-induced degradation of PIF1. J Biol Chem, 286, 12066-12074.

Castillon, A., Shen, H. and Huq, E. (2009) Blue light induces degradation of the negative regulator Phytochrome Interacting Factor 1 to promote photomorphogenic development of Arabidopsis seedlings. Genetics, 182, 161171.

Chen, H., Shen, Y., Tang, X., Yu, L., Wang, J., Guo, L., Zhang, Y., Zhang, H., Feng, S., Strickland, E., et al (2006) Arabidopsis CULLIN4 Forms an E3 Ubiquitin Ligase with RBX1 and the CDD Complex in Mediating Light Control of Development. Plant Cell, 18, 1991-2004.

Deng, X.-W., Matsui, M., Wei, N., Wagner, D., Chu, A. M., Feldman, K. A. and Quail, P. H. (1992) COP1, an arabidopsis regulatory gene, encodes a protein with both a zinc-binding motif and a G $\beta$ homologous domain. Cell, 71, 791-801.

Dong, J., Ni, W., Yu, R., Deng, X. W., Chen, H. and Wei, N. (2017) Light-Dependent Degradation of PIF3 by $\mathrm{SCF}<\mathrm{sup}>\mathrm{EBF} 1 / 2</$ sup $>$ Promotes a Photomorphogenic Response in <em>Arabidopsis</em>. Current Biology, 27, 2420-2430.

Dong, J., Tang, D., Gao, Z., Yu, R., Li, K., He, H., Terzaghi, W., Deng, X. W. and Chen, H. (2014) Arabidopsis DE-ETIOLATED1 Represses Photomorphogenesis by Positively Regulating Phytochrome-Interacting Factors in the Dark. The Plant Cell Online, 26, 3630-3645.

Fierro, A. C., Leroux, O., De Coninck, B., Cammue, B. P. A., Marchal, K., Prinsen, E., Van Der Straeten, D. and Vandenbussche, F. (2015) Ultraviolet-B radiation stimulates downward leaf curling in Arabidopsis thaliana. Plant Physiology and Biochemistry, 93, 9-17. 
Gommers, C. M. M. and Monte, E. (2018) Seedling establishment: a dimmer switchregulated process between dark and light signaling. Plant Physiol, 176, 10611074.

Gu, Z., Eils, R. and Schlesner, M. (2016) Complex heatmaps reveal patterns and correlations in multidimensional genomic data. Bioinformatics, 32, 2847-2849.

Hardtke, C. S., Gohda, K., Osterlund, M. T., Oyama, T., Okada, K. and Deng, X. W. (2000) HY5 stability and activity in arabidopsis is regulated by phosphorylation in its COP1 binding domain. Embo J, 19, 4997-5006.

Hoecker, U. (2017) The activities of the E3 ubiquitin ligase COP1/SPA, a key repressor in light signaling. Current Opinion in Plant Biology, 37, 63-69.

Hornitschek, P., Lorrain, S., Zoete, V., Michielin, O. and Fankhauser, C. (2009) Inhibition of the shade avoidance response by formation of non-DNA binding bHLH heterodimers. Embo J, 28, 3893-3902.

Huang, X., Ouyang, X., Yang, P., Lau, O. S., Chen, L., Wei, N. and Deng, X. W. (2013) Conversion from CUL4-based COP1-SPA E3 apparatus to UVR8-COP1SPA complexes underlies a distinct biochemical function of COP1 under UV-B. Proceedings of the National Academy of Sciences, 110, 16669-16674.

Huq, E., Al-Sady, B., Hudson, M., Kim, C., Apel, K. and Quail, P. H. (2004) Phytochrome-interacting factor 1 is a critical bHLH regulator of chlorophyll biosynthesis. Science, 305, 1937-1941.

Huq, E. and Quail, P. H. (2002) PIF4, a phytochrome-interacting bHLH factor, functions as a negative regulator of phytochrome B signaling in Arabidopsis. Embo J, 21, 2441-2450.

Jang, I. C., Yang, J. Y., Seo, H. S. and Chua, N. H. (2005) HFR1 is targeted by COP1 E3 ligase for post-translational proteolysis during phytochrome A signaling. Genes Dev, 19, 593-602.

Khanna, R., Shen, Y., Marion, C. M., Tsuchisaka, A., Theologis, A., Schäfer, E. and Quail, P. H. (2007) The Basic Helix-Loop-Helix Transcription Factor PIF5 Acts on Ethylene Biosynthesis and Phytochrome Signaling by Distinct Mechanisms. Plant Cell, 19, 3915-3929.

Langmead, B. and Salzberg, S. L. (2012) Fast gapped-read alignment with Bowtie 2. Nature Methods, 9, 357.

Lau, O. S. and Deng, X. W. (2012) The photomorphogenic repressors COP1 and DET1: 20 years later. Trends Plant Sci, 17, 584-593.

Laubinger, S., Fittinghoff, K. and Hoecker, U. (2004) The SPA Quartet: A Family of WD-Repeat Proteins with a Central Role in Suppression of Photomorphogenesis in Arabidopsis. Plant Cell.

Leivar, P., Monte, E., Oka, Y., Liu, T., Carle, C., Castillon, A., Huq, E. and Quail, P. H. (2008) Multiple phytochrome-interacting bHLH transcription factors repress premature seedling photomorphogenesis in darkness. Curr Biol, 18, 1815-1823. 
Leivar, P. and Quail, P. H. (2011) PIFs: pivotal components in a cellular signaling hub. Trends Plant Sci, 16, 19-28.

Love, M. I., Huber, W. and Anders, S. (2014) Moderated estimation of fold change and dispersion for RNA-seq data with DESeq2. Genome Biology, 15, 550.

Monte, E., Tepperman, J. M., Al-Sady, B., Kaczorowski, K. A., Alonso, J. M., Ecker, J. R., Li, X., Zhang, Y. and Quail, P. H. (2004) The phytochrome-interacting transcription factor, PIF3, acts early, selectively, and positively in light-induced chloroplast development. Proc Natl Acad Sci U S A, 101, 16091-16098.

Moon, J., Zhu, L., Shen, H. and Huq, E. (2008) PIF1 directly and indirectly regulates chlorophyll biosynthesis to optimize the greening process in Arabidopsis. Proc Natl Acad Sci U S A, 105, 9433-9438.

Ni, W., Xu, S.-L., González-Grandío, E., Chalkley, R. J., Huhmer, A. F. R., Burlingame, A. L., Wang, Z.-Y. and Quail, P. H. (2017) PPKs mediate direct signal transfer from phytochrome photoreceptors to transcription factor PIF3. Nature Communications, 8, 15236.

Ni, W., Xu, S.-L., Tepperman, J. M., Stanley, D. J., Maltby, D. A., Gross, J. D., Burlingame, A. L., Wang, Z.-Y. and Quail, P. H. (2014) A mutually assured destruction mechanism attenuates light signaling in Arabidopsis. Science, 344, 1160-1164.

Oh, E., Kim, J., Park, E., Kim, J. I., Kang, C. and Choi, G. (2004) PIL5, a phytochrome-interacting basic helix-loop-helix protein, is a key negative regulator of seed germination in Arabidopsis thaliana. Plant Cell, 16, 3045-3058.

Osterlund, M. T., Hardtke, C. S., Wei, N. and Deng, X. W. (2000) Targeted destabilization of HY5 during light-regulated development of Arabidopsis. Nature, 405, 462-466.

Paik, I., Kathare, P. K., Kim, J.-I. and Huq, E. (2017) Expanding Roles of PIFs in Signal Integration from Multiple Processes. Mol Plant, 10, 1035-1046.

Park, E., Kim, J., Lee, Y., Shin, J., Oh, E., Chung, W. I., Liu, J. R. and Choi, G. (2004) Degradation of phytochrome interacting factor 3 in phytochrome-mediated light signaling. Plant Cell Physiol, 45, 968-975.

Pepper, A., Delaney, T., Washburnt, T., Poole, D. and Chory, J. (1994) DET1, a negative regulator of light-mediated development and gene expression in arabidopsis, encodes a novel nuclear-localized protein. Cell, 78, 109-116.

Pfeiffer, A., Shi, H., Tepperman, J. M., Zhang, Y. and Quail, P. H. (2014) Combinatorial Complexity in a Transcriptionally-centered Signaling Hub in Arabidopsis. Mol Plant, 7, 1598-1618.

Pham, V. N., Hoecker, U. and Huq, E. (2018a) Genomic evidence reveals SPAregulated developmental and metabolic pathways in dark-grown Arabidopsis seedlings. BMC Genomics, Submitted.

Pham, V. N., Kathare, P. K. and Huq, E. (2018b) Phytochromes and Phytochrome Interacting Factors. Plant Physiology, 176, 1025-1038. 
Saijo, Y., Sullivan, J. A., Wang, H., Yang, J., Shen, Y., Rubio, V., Ma, L., Hoecker, U. and Deng, X. W. (2003) The COP1-SPA1 interaction defines a critical step in phytochrome A-mediated regulation of HY5 activity. Genes Dev, 17, 2642-2647.

Sakuraba, Y., Jeong, J., Kang, M.-Y., Kim, J., Paek, N.-C. and Choi, G. (2014) Phytochrome-interacting transcription factors PIF4 and PIF5 induce leaf senescence in Arabidopsis. Nature communications, 5, 4636.

Schroeder, D. F., Gahrtz, M., Maxwell, B. B., Cook, R. K., Kan, J. M., Alonso, J. M., Ecker, J. R. and Chory, J. (2002) De-Etiolated 1 and Damaged DNA Binding Protein 1 Interact to Regulate Arabidopsis Photomorphogenesis. Current Biology, 12, 1462-1472.

Seo, H. S., Yang, J. Y., Ishikawa, M., Bolle, C., Ballesteros, M. L. and Chua, N. H. (2003) LAF1 ubiquitination by COP1 controls photomorphogenesis and is stimulated by SPA1. Nature, 423, 995-999.

Serino, G. and Deng, X.-W. (2003) The COP9 signalosome: regulating plant development through the control of proteolysis. Annu. Rev. Plant Biol., 54, 165182.

Shen, H., Ling, Z., Castillon, A., Majee, M., Downie, B. and Huq, E. (2008) Lightinduced phosphorylation and degradation of the negative regulator PHYTOCHROME INTERACTING FACTOR 1 depends upon its direct physical interactions with photoactivated phytochromes. Plant Cell, 20, 1586-1602.

Shen, H., Moon, J. and Huq, E. (2005) PIF1 is regulated by light-mediated degradation through the ubiquitin-26S proteasome pathway to optimize seedling photomorphogenesis in Arabidopsis. Plant J, 44, 1023-1035.

Shi, H., Wang, X., Mo, X., Tang, C., Zhong, S. and Deng, X. W. (2015) Arabidopsis DET1 degrades HFR1 but stabilizes PIF1 to precisely regulate seed germination. Proceedings of the National Academy of Sciences, 112, 3817-3822.

Shi, H., Zhong, S., Mo, X., Liu, N., Nezames, C. D. and Deng, X. W. (2013) HFR1 Sequesters PIF1 to Govern the Transcriptional Network Underlying LightInitiated Seed Germination in Arabidopsis. The Plant Cell, 25, 3770-3784.

Shin, J., Kim, K., Kang, H., Zulfugarov, I. S., Bae, G., Lee, C. H., Lee, D. and Choi, G. (2009) Phytochromes promote seedling light responses by inhibiting four negatively-acting phytochrome-interacting factors. Proc. Nat. Acad. Sci. U S A, 106, 7660-7665.

Shor, E., Paik, I., Kangisser, S., Green, R. and Huq, E. (2017) PHYTOCHROME INTERACTING FACTORS mediate metabolic control of the circadian system in Arabidopsis. New Phytologist, 215, 217-228.

Stephenson, P. G., Fankhauser, C. and Terry, M. J. (2009) PIF3 is a repressor of chloroplast development. Proc Natl Acad Sci U S A, 106, 7654-7659.

Tilbrook, K., Arongaus, A. B., Binkert, M., Heijde, M., Yin, R. and Ulm, R. (2013) The UVR8 UV-B Photoreceptor: Perception, Signaling and Response. The Arabidopsis Book, e0164. 
Toledo-Ortíz, G., Huq, E. and Rodríguez-Concepción, M. (2010) Direct regulation of phytoene synthase gene expression and carotenoid biosynthesis by PhytochromeInteracting Factors. Proc Natl Acad Sci U S A, 107, 11626-11631.

Trapnell, C., Roberts, A., Goff, L., Pertea, G., Kim, D., Kelley, D. R., Pimentel, H., Salzberg, S. L., Rinn, J. L. and Pachter, L. (2012) Differential gene and transcript expression analysis of RNA-seq experiments with TopHat and Cufflinks. Nature protocols, 7, 562-578.

Xu, X., Kathare, P. K., Pham, V. N., Bu, Q., Nguyen, A. and Huq, E. (2017) Reciprocal proteasome-mediated degradation of PIFs and HFR1 underlies photomorphogenic development in <em $>$ Arabidopsis</em $>$. Development, 144, 1831-1840.

Xu, X., Paik, I., Zhu, L., Bu, Q., Huang, X., Deng, X. W. and Huq, E. (2014) PHYTOCHROME INTERACTING FACTOR1 Enhances the E3 Ligase Activity of CONSTITUTIVE PHOTOMORPHOGENIC1 to Synergistically Repress Photomorphogenesis in Arabidopsis. Plant Cell, 26, 1992-2006.

Xu, X., Paik, I., Zhu, L. and Huq, E. (2015) Illuminating Progress in PhytochromeMediated Light Signaling Pathways. Trends in plant science, 20, 641-650.

Yang, J., Lin, R., Hoecker, U., Liu, B., Xu, L. and Wang, H. (2005a) Repression of Light Signaling by Arabidopsis SPA1 Involves Post-translational Regulation of HFR1 Protein Accumulation. . Plant J, 43, 131-141.

Yang, J., Lin, R., Sullivan, J., Hoecker, U., Liu, B., Xu, L., Deng, X. W. and Wang, H. (2005b) Light Regulates COP1-Mediated Degradation of HFR1, a Transcription Factor Essential for Light Signaling in Arabidopsis. Plant Cell, 17, 804-821.

Zhang, B., Holmlund, M., Lorrain, S., Norberg, M., Bakó, L., Fankhauser, C. and Nilsson, O. (2017) BLADE-ON-PETIOLE proteins act in an E3 ubiquitin ligase complex to regulate PHYTOCHROME INTERACTING FACTOR 4 abundance. eLife, 6, e26759.

Zhang, Y., Mayba, O., Pfeiffer, A., Shi, H., Tepperman, J. M., Speed, T. P. and Quail, P. H. (2013) A Quartet of PIF bHLH Factors Provides a Transcriptionally Centered Signaling Hub That Regulates Seedling Morphogenesis through Differential Expression-Patterning of Shared Target Genes in Arabidopsis. Plos Genetics, 9, e1003244.

Zhu, D. M., Maier, A., Lee, J.-H., Laubinger, S., Saijo, Y., Wang, H. Y., Qu, L.-J., Hoecker, U. and Deng, X. W. (2008) Biochemical Characterization of Arabidopsis Complexes Containing CONSTITUTIVELY PHOTOMORPHOGENIC1 and SUPPRESSOR OF PHYA Proteins in Light Control of Plant Development. Plant Cell, 20, 2307-2323.

Zhu, L., Bu, Q., Xu, X., Paik, I., Huang, X., Hoecker, U., Deng, X. W. and Huq, E. (2015) CUL4 forms an E3 ligase with COP1 and SPA to promote light-induced degradation of PIF1. Nature communications, $\mathbf{6}, 7245$. 
Zhu, L. and Huq, E. (2014) Suicidal co-degradation of the Phytochrome Interacting Factor 3 and phytochrome B in response to light. Mol Plant, 7, 1709-1711.

Zhu, L., Xin, R., Bu, Q., Shen, H., Dang, J. and Huq, E. (2016) A negative feedback loop between PHYTOCHROME INTERACTING FACTORs and HECATE proteins fine tunes photomorphogenesis in Arabidopsis. Plant Cell, 28, 855-874. 


\section{Figure Legends:}

\section{Figure 1: COP1 positively regulates PIF protein level in darkness.}

(A) Visible constitutive photomorphogenic phenotypes of 4-day-old dark grown seedlings. Bar $=10 \mathrm{~mm}$. (B) Immunoblot showing COP1 endogenous protein level in wild-type (Col-0), cop1-4, spaQ and pifQ dark-grown seedlings. Total protein was extracted from 4-day old dark-grown seedlings, separated on 8\% SDS-PAGE gel, probed with anti-COP1 and anti-RPT5 antibody. (C-F) Immunoblots showing PIFs protein level and quantitative graphs. The overexpression plants TAP-PIF1, PIF3-Myc, PIF4-Myc and PIF5-Myc in Col-0 and cop1-4 backgrounds were grown under the condition described above. Total protein was separated in 6.5\% SDS-PAGE gel and probed with anti-Myc and anti-RPT5 antibodies. PIFs protein level were quantified from three biological replicates $(n=3)$ and normalized with RPT5 levels. The PIF protein level in Col-0 was set as 1 . * indicates $\mathrm{P}<0.05$, ** indicates $\mathrm{P}<0.01$.

Figure 2: Instability of PIFs in the dark in cop1 backgrounds is 26S-proteasome dependent

(A) PIF protein levels in 4-day-old dark-grown wild-type and cop1-4 seedlings with and without treatment with 26S protease inhibitor (Bortezomib or Bortz). Total proteins were extracted and separated on 6.5\% SDS-PAGE and probed with anti-Myc and anti-RPT5 antibodies. RPT5 was used as loading control. (B) Bar graphs showing the quantitative PIF protein levels in those backgrounds from three biological replicates $(n=3)$. In each graph, PIF protein level in Col-0 background without Bortezomib treatment was set as 1 . Error bars indicate mean $\pm \mathrm{SD}$. * indicates $\mathrm{P}<0.05$.

\section{Figure 3: Overexpression PIFs partially suppresses the constitutive photomorphogenetic phenotypes of cop1-4.}

(A-B) Visible phenotypes of the 4-day-old dark-grown PIF overexpression in Col-0 and cop1-4 backgrounds. White bar $=10 \mathrm{~mm}$. (C) Box plots represent the hypocotyl lengths and cotyledon opening angle measurements. Three independent biological replicates were performed with an average of 30 PIF overexpression in Col-0 and cop1-4 seedlings 
grown under the same conditions as described above. Significant difference between different genotypes was determined using one-way ANOVA and Tukey's HSD tests, indicated by different letters.

\section{Figure 4: COP1 and SPA mediate light-regulated transcriptomic changes partly through PIFs.}

(A) Venn diagram showing 1120 co-regulated, 483 co-upregulated and 431 codownregulated differential expressed genes in 3 different pairwise comparisons indicated (cop1-4/WT, spaQ/WT and pifQ/WT). (B)Hierarchical clustering displays 1120 DEGs in comparisons indicated. Data showing genes co-regulated identified as at least 2-fold difference in gene expression $(\mathrm{FDR}<0.05)$ The color represents the $\log 2$ of fold change. cop 1-4/WT and spaQ/WT: comparison of the expression profiles of dark-grown cop1-4 and spaQ with Col-0, respectively. (C, D) Bar graphs showing Enrichment analysis of Gene Ontology Biological Processes significantly co-upregulated (C) and codownregulated (D) in copl-4/WT, spaQ/WT, and $p i f Q / W T$. Enrichment scores indicate percentages, involved genes/total genes. Fisher Exact P-Values were presented on the top of each bar.

\section{Figure 5: PIF-direct target genes are co-regulated in cop1-4 and spaQ mutants.}

(A) Venn diagram showing among 338 PIF-direct target genes, 170 genes are coregulated by COP1 and SPA, 110 genes are down- regulated and 42 genes are upregulated in copl-4 and spaQ mutants. (B) to (C) qRT-PCR shows the similar expression patterns of various PIF-induced genes (B) and PIF-repressed genes (C) in copl-4, spaQ and pifQ in the dark. Gene expression level in mutants were normalized to $P P 2 A$ and expression level in Col-0 was set as 1. (D) To (E) Expression patterns of various PIFinduced genes (D) and PIF-repressed genes (E) in copl-4, spaQ and pifQ in the dark from RNA-seq data.

Figure 6: The transcriptional activation activity of PIFs is higher in cop1-4hfrl compared to cop1-4 background 
(A) The expression levels of PIF target genes are lower in the pifQ and copl-4 background, but are higher in copl-4 hfrl background. Total seedling RNA was extracted from 3-day-old dark grown seedlings of wild type Col-0, pifQ, copl-4 and copl-4 hfrl. $P P 2 A$ was used as an internal control. Wild type Col-0 was set as 1. Error bars indicate standard deviation ( $\mathrm{n}=3$ independent biological repeats). (B) The PILI and XTR7 promoter occupancies of TAP-PIF1 were reduced in copl-4 background but increased in cop1-4hfrl background. The ChIP-qPCR assays were performed on 3-day-old dark grown seeding expressing TAP-PIF1 fusion protein in copl-4 and copl-4hfrl backgrounds. (C)Photographs of seedlings of wild type, copl-4, copl-4hfrl, copl4/TAP-PIF1 and cop1-4hfrl/TAP-PIF1. Seedlings were grown in the dark for 5 days. White bar $=5 \mathrm{~mm}$. (D) Bar graph showing hypocotyl lengths of various genotypes as described in (C). Error bars indicate standard deviation. Significant difference between different genotypes was determined using one-way ANOVA and Tukey's HSD tests, indicated by different letters.

\section{Figure 7: Model showing how COP1 and SPA proteins regulate various} transcription factors to promote skotomorphogenesis in the dark. Mutation in COP1, SPA and PIFs results in constitutive photomophogenic (cop) phenotypes in the dark. PIFs and HFR1 reciprocally regulate their abundance, while HFR1 inhibits the PIF activity by sequestration. Regulation of these transcription factor abundance and activity by the COP1-SPA complex promotes skotomorphogenic development. 
A

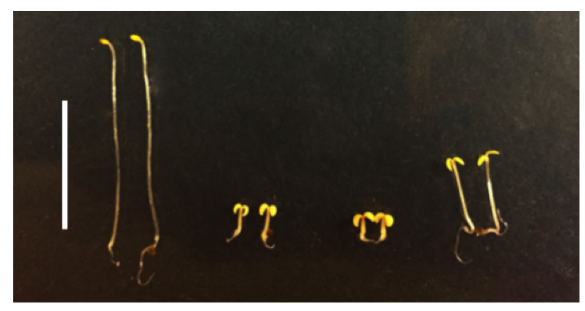

Col-0 cop1-4 spaQ pifQ

C

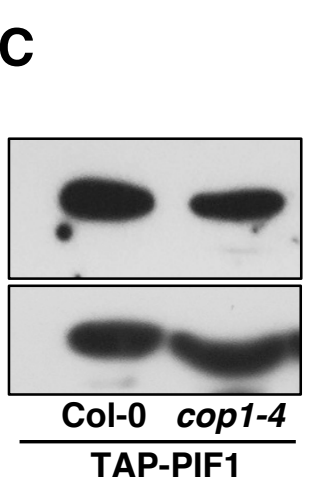

E

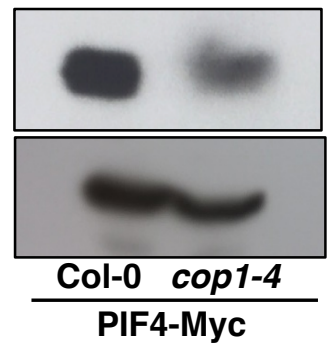

B
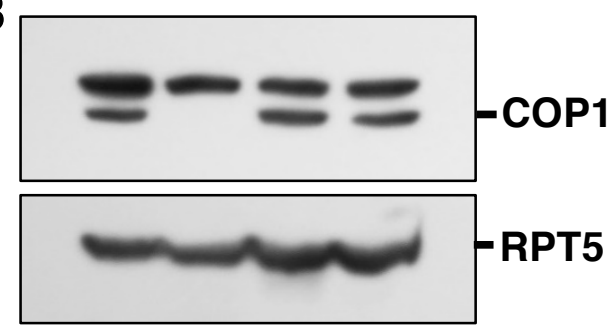

$c 01-0$ copt $s p^{1-A} p i f^{Q}$
RPT5

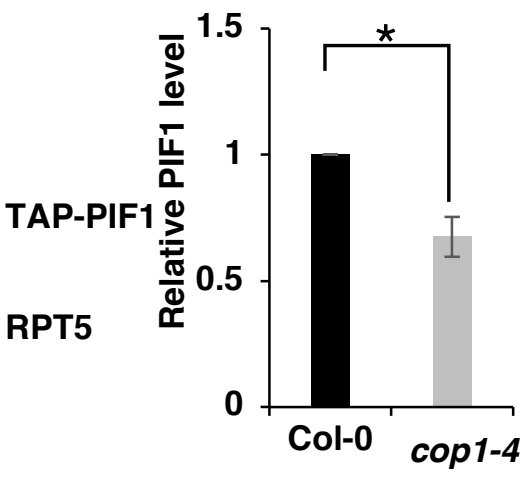

PIF4-Myc

RPT5
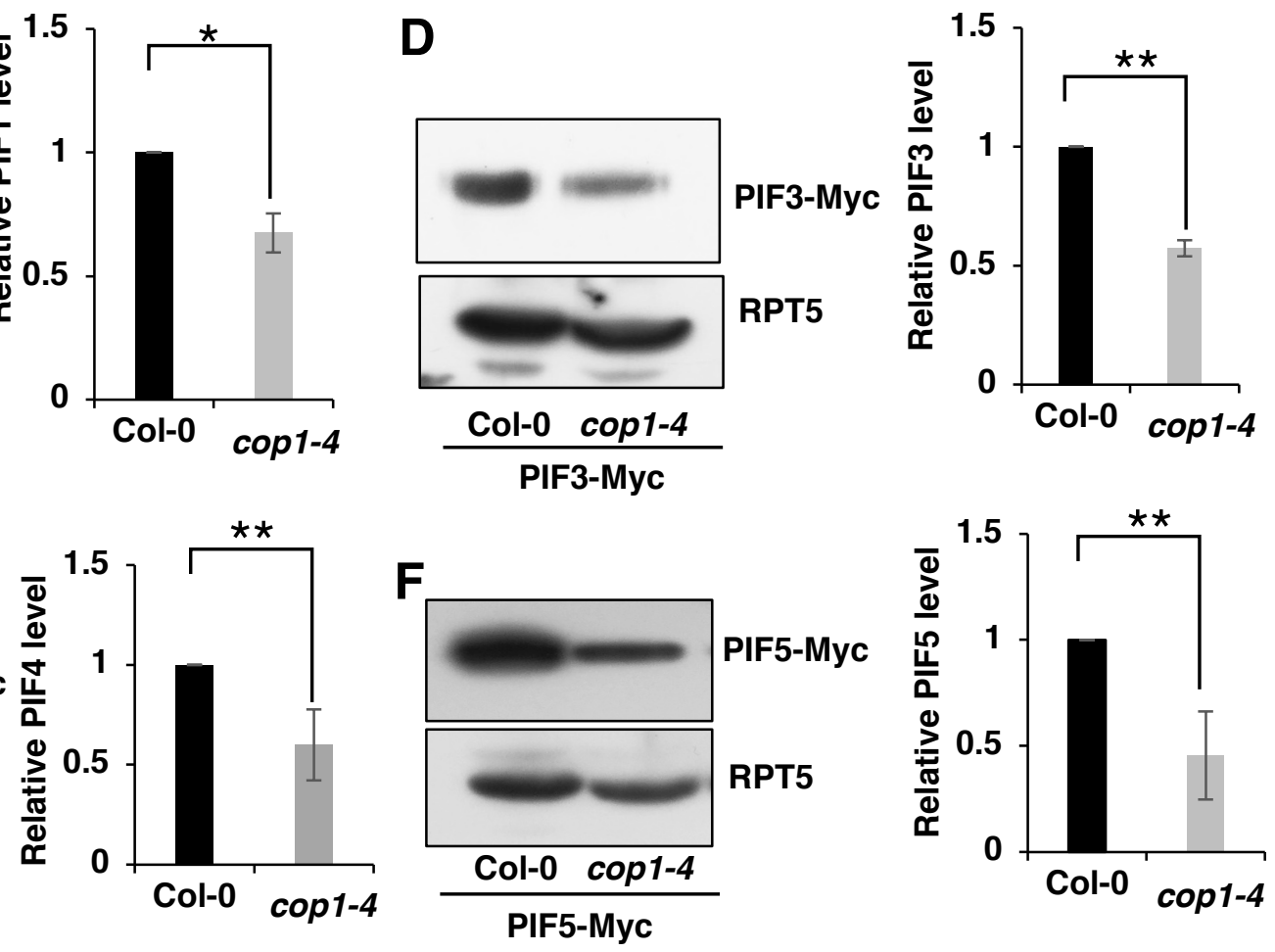

Figure 1: COP1 positively regulates PIF protein level in darkness.

(A) Visible constitutive photomorphogenic phenotypes of 4-day-old dark grown seedlings. Bar $=10 \mathrm{~mm}$. (B) Immunoblot showing COP1 endogenous protein level in wild-type (Col-0), cop1-4, spaQ and pifQ dark-grown seedlings. Total protein was extracted from 4-day old dark-grown seedlings, separated on $8 \%$ SDS-PAGE gel, probed with anti-COP1 and antiRPT5 antibody. (C-F) Immunoblots showing PIFs protein level and quantitative graphs. The overexpression plants TAP-PIF1, PIF3-Myc, PIF4-Myc and PIF5-Myc in Col-0 and cop1-4 backgrounds were grown under the condition described above. Total protein was separated in $6.5 \%$ SDS-PAGE gel and probed with anti-Myc and anti-RPT5 antibodies. PIFs protein level were quantified from three biological replicates $(n=3)$ and normalized with RPT5 levels. The PIF protein level in Col-0 was set as $1 .{ }^{*}$ indicates $P<0.05,{ }^{* *}$ indicates $P<$ 0.01 . 


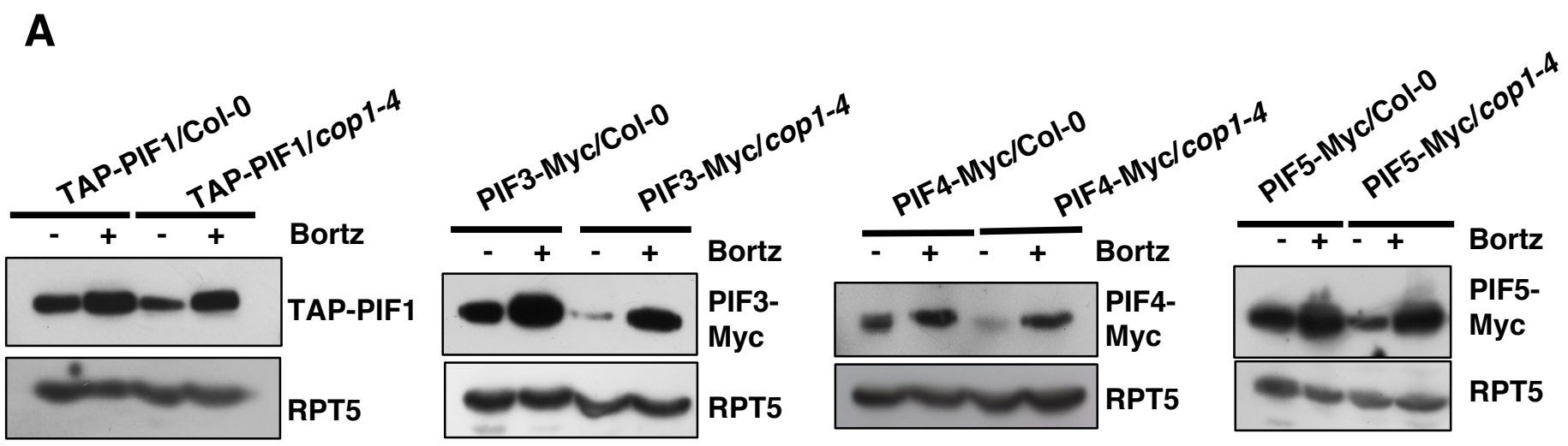

\section{B}
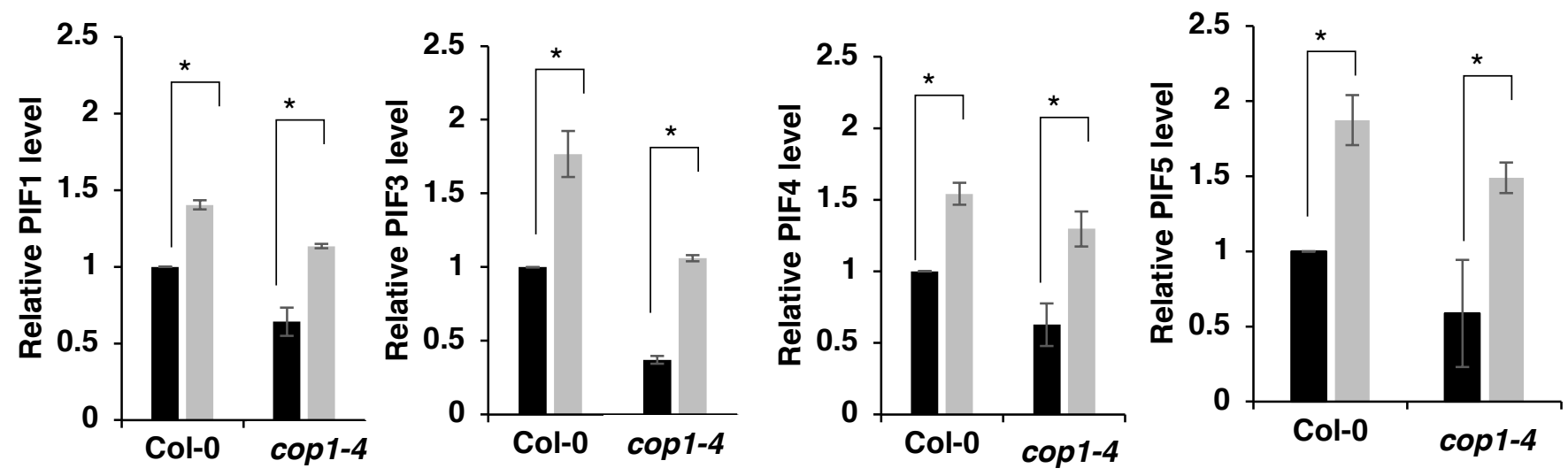

-Without Bortezomib

With Bortezomib

Figure 2: Instability of PIFs in the dark in cop1 backgrounds is 26S-proteasome dependent.

(A) PIF protein levels in 4-day-old dark-grown wild-type and cop1-4 seedlings with and without treatment with $26 \mathrm{~S}$ protease inhibitor (Bortezomib or Bortz). Total proteins were extracted and separated on $6.5 \%$ SDS-PAGE and probed with anti-Myc and anti-RPT5 antibodies. RPT5 was used as loading control. (B) Bar graphs showing the quantitative PIF protein levels in those backgrounds from three biological replicates $(n=3)$. In each graph, PIF protein level in Col-0 background without Bortezomib treatment was set as 1 . Error bars indicate mean $\pm \mathrm{SD}$. * indicates $P<0.05$. 
bioRxiv preprint doi: https://doi.org/10.1101/388157; this version posted August 9, 2018. The copyright holder for this preprint (which was not

A
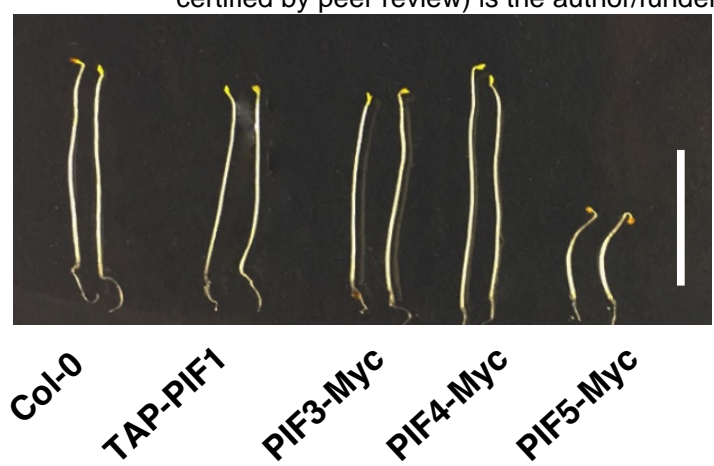

C

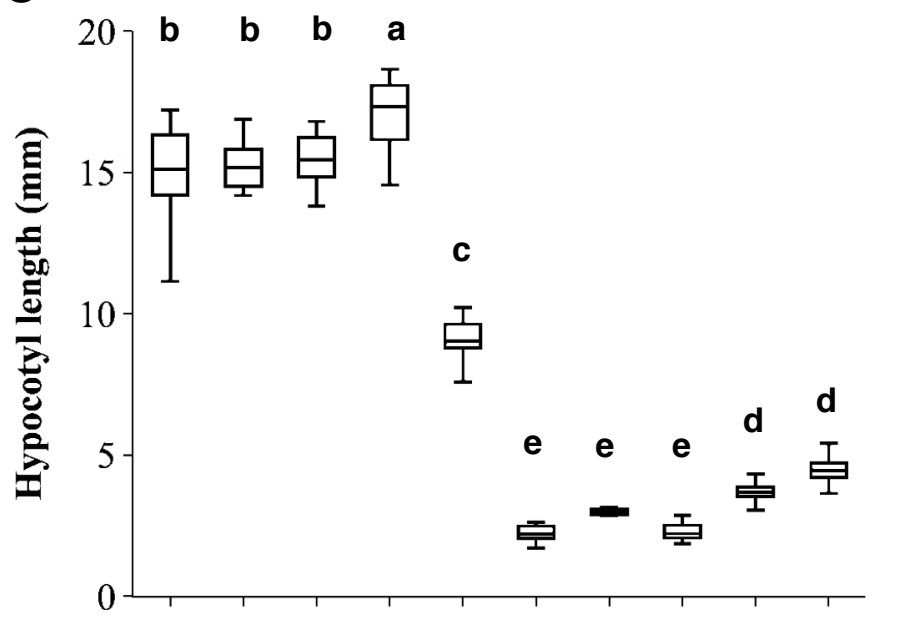

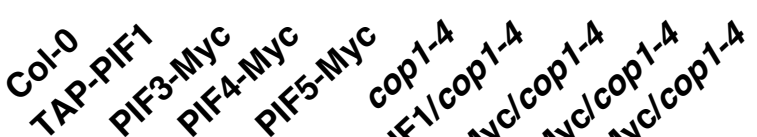

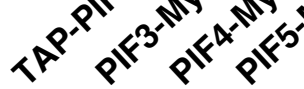

B
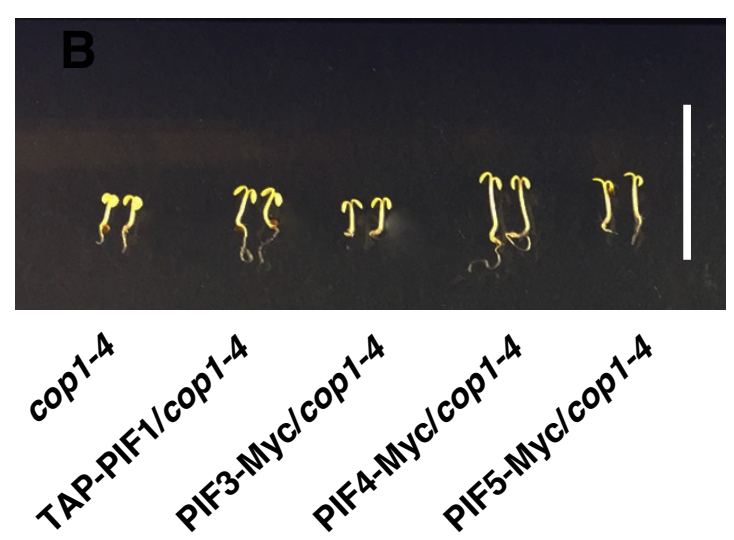

D

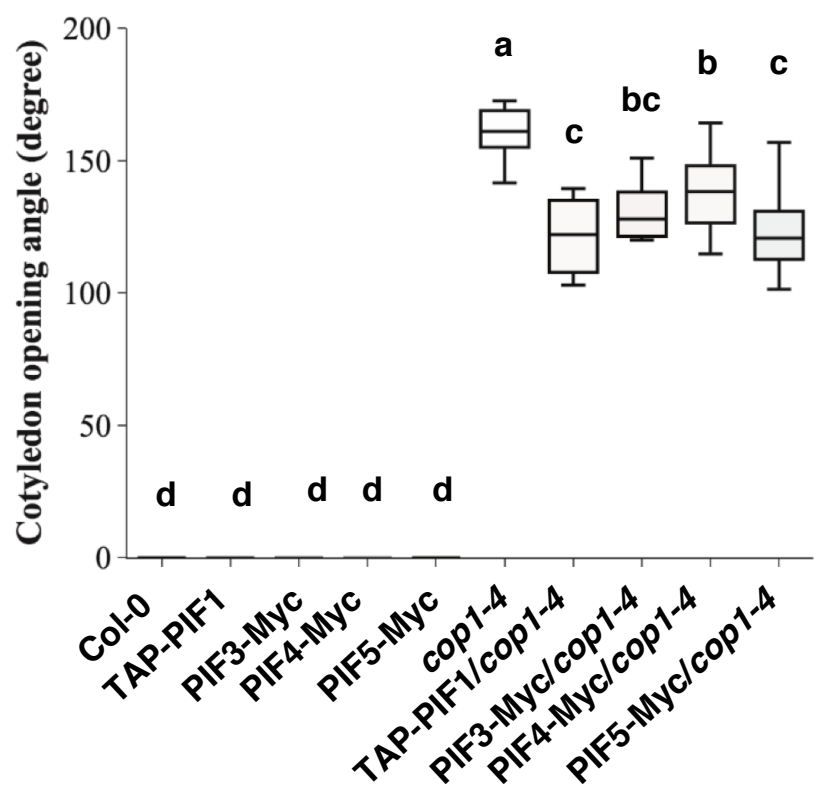

Figure 3: Overexpression PIFs partially suppresses the constitutive photomorphogenetic phenotypes of cop1-4.

(A-B) Visible phenotypes of the 4-day-old dark-grown PIF overexpression in Col-0 and cop1-4 backgrounds. White bar $=10 \mathrm{~mm}$. (C) Box plots represent the hypocotyl lengths and cotyledon opening angle measurements. Three independent biological replicates were performed with an average of 30 PIF overexpression in Col-0 and cop1-4 seedlings grown under the same conditions as described above. Significant difference between different genotypes was determined using one-way ANOVA and Tukey's HSD tests, indicated by different letters. 
bioRxiv preprint doi: https://doi.org/10.1101/388157; this version posted August 9, 2018. The copyright holder for this preprint (which was not

A certified by peer review) is the author/funder. All rights reserved. No reuse allowed without permission.

cop1-4/WT regulated genes

(5249)

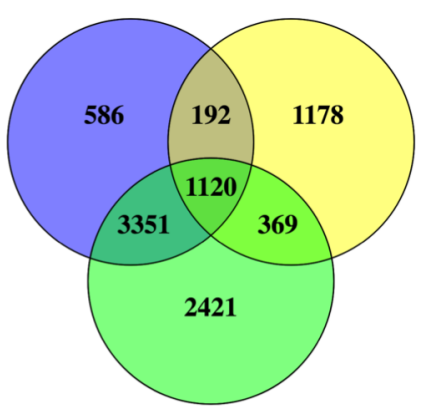

spaQ/WT regulated genes

(7261)
pifq/WT regulated genes

(2859)

B

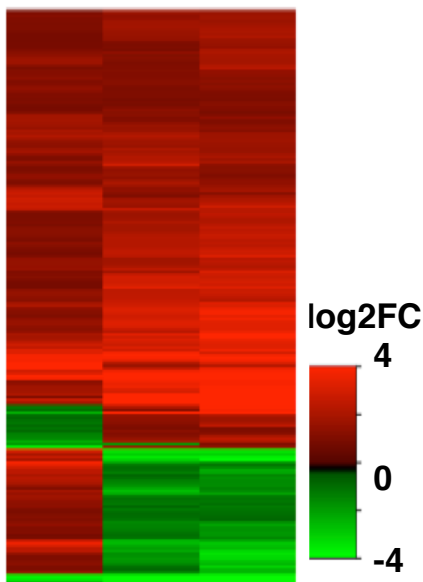

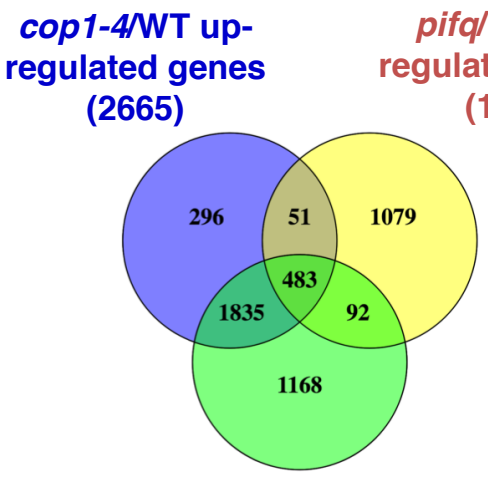

spaQ/WT up-regulated genes ( 3578)

(1705)
cop1-4/WT down-
regulated genes

( 2584)
pifg/WT down-

regulated genes

(1154)

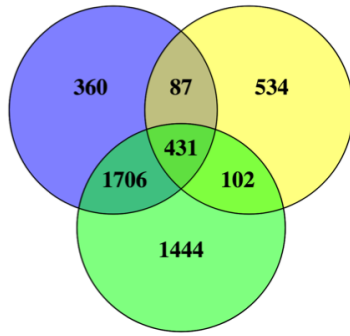

spaQ/WT down-regulated genes

(3684)
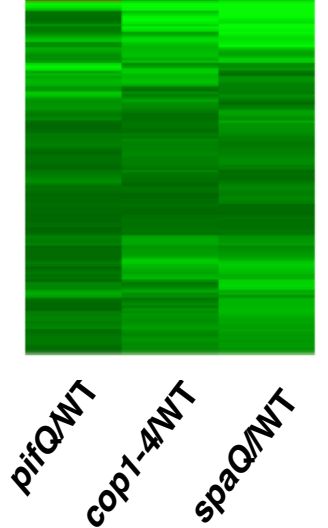
A cop1-4/WT regulated genes PIF-direct target genes (338)
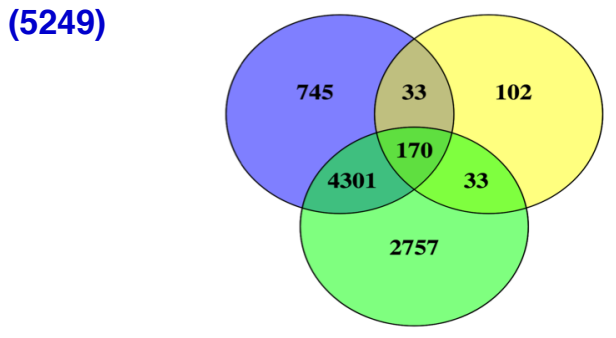

spaQ/WT regulated genes

(7261)

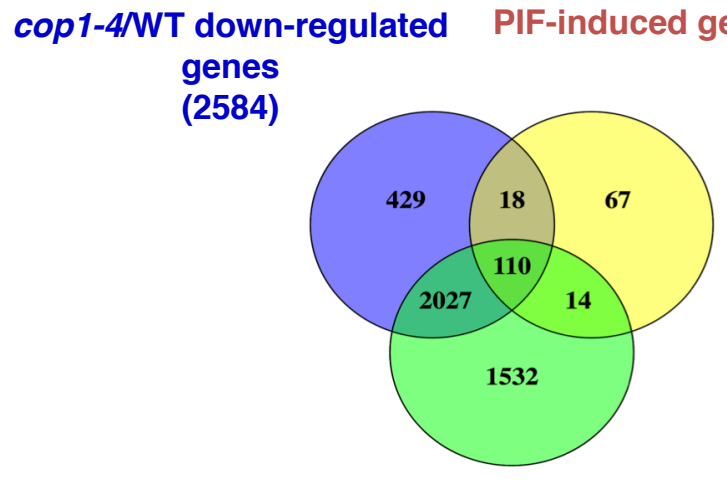

spaQ/WT down-regulated genes

(3684)
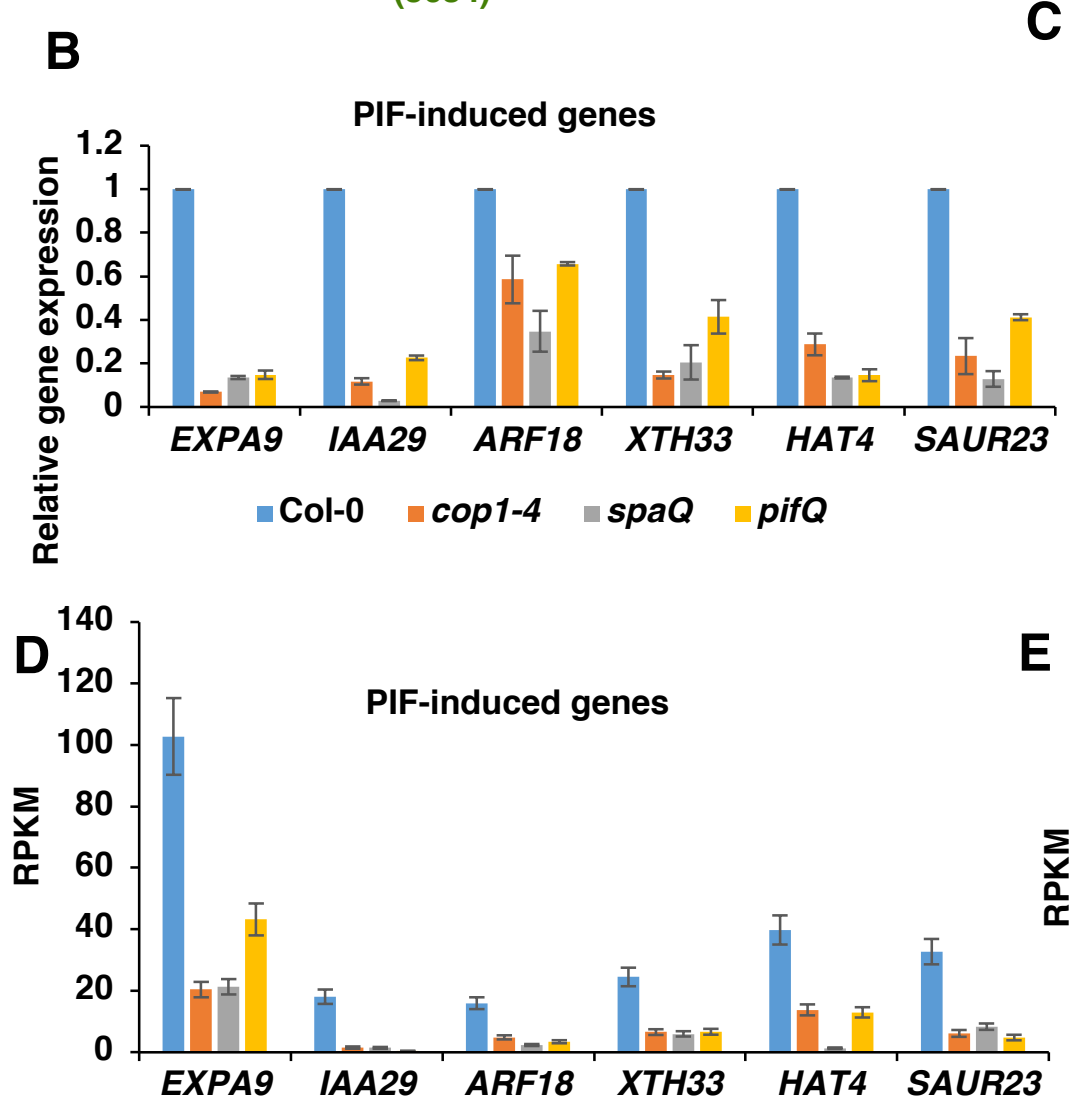

E
cop1-4/WT upregulated genes (2665)

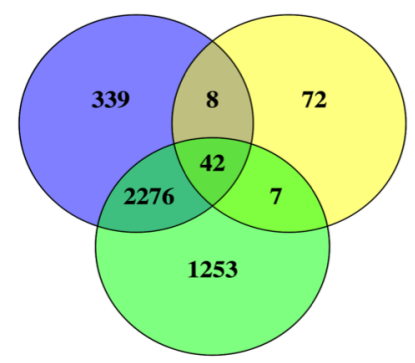

spaQ/WT up-regulated genes

(3578)

C

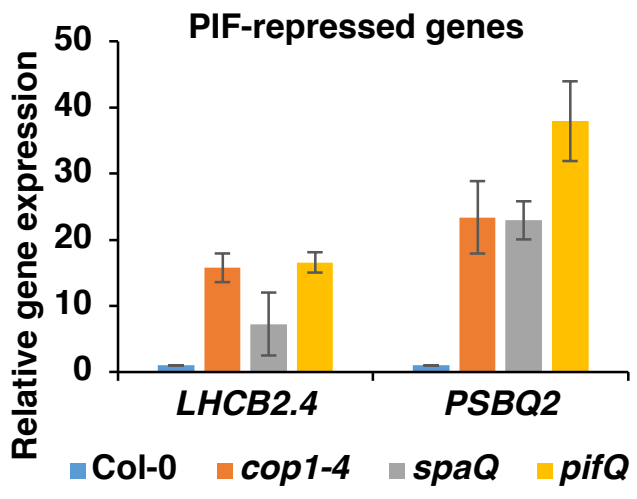

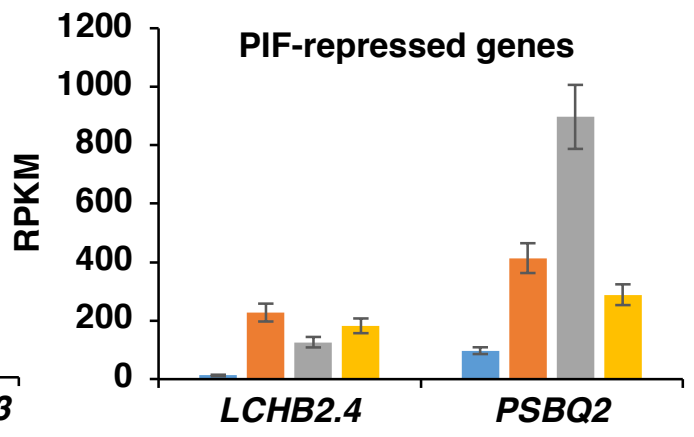

Figure 5: PIF-direct target genes are co-regulated in cop1-4 and spaQ mutants.

(A) Venn diagram showing among 338 PIF-direct target genes, 170 genes are co-regulated by COP1 and SPA, 110 genes are down- regulated and 42 genes are up-regulated in cop1-4 and spaQ mutants. (B) to (C) qRT-PCR shows the similar expression patterns of various PIF-induced genes (B) and PIF-repressed genes $(C)$ in cop 1-4, spaQ and pifQ in the dark. Gene expression level in mutants were normalized to $P P 2 A$ and expression level in Col-0 was set as 1. (D) To (E) Expression patterns of various PIF-induced genes (D) and PIF-repressed genes (E) in cop1-4, spaQ and pifQ in the dark from RNA-seq data. 
A certified by peer review) is the author/funder. All rights reserved. No reuse allowed without permission.
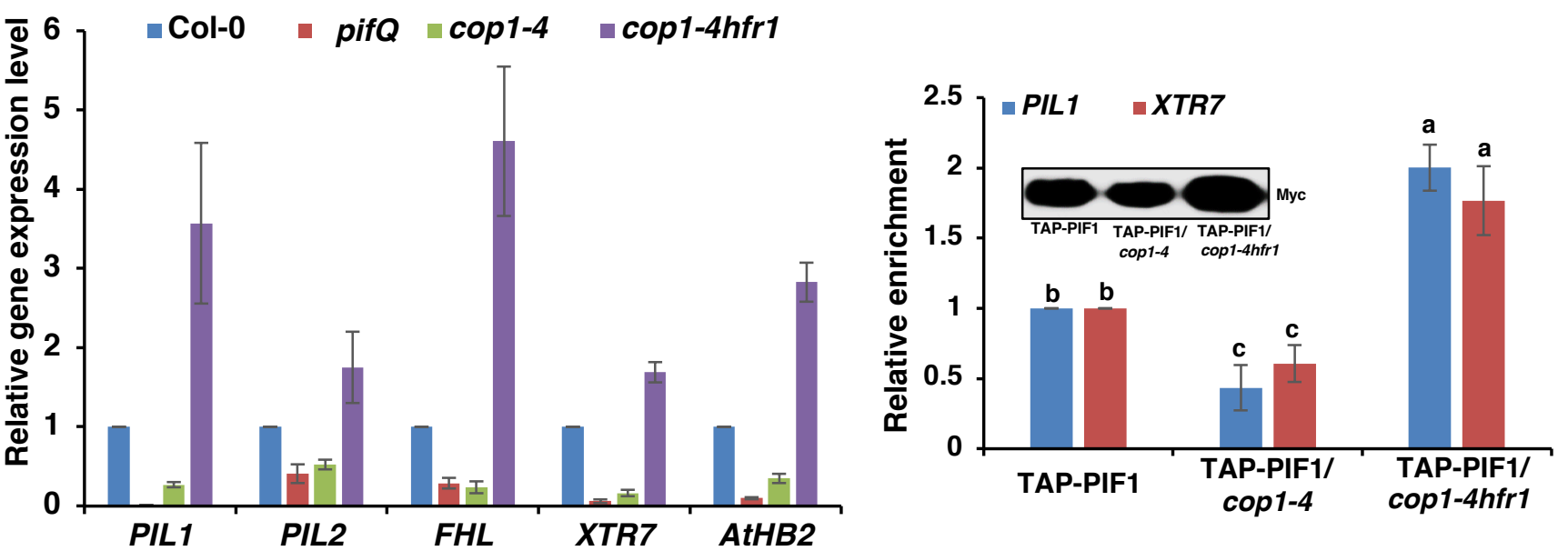

C
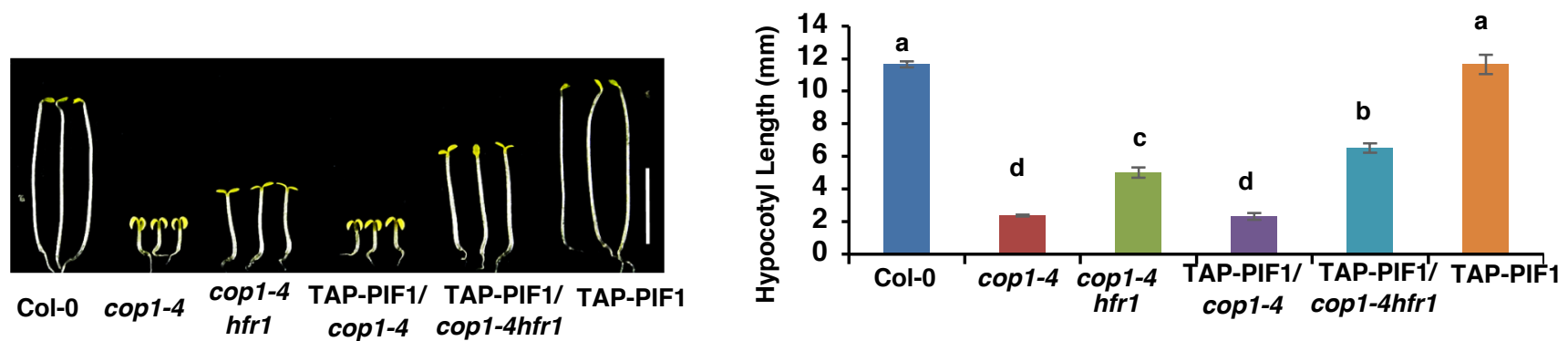

Figure 6: The transcriptional activation activity of PIFs is higher in cop1-4hfr1 compared to cop1-4 background

(A) The expression levels of PIF target genes are lower in the pifQ and cop1-4 background, but are higher in cop1-4 hfr1 background. Total seedling RNA was extracted from 3-day-old dark grown seedlings of wild type Col-0, pifQ, cop1-4 and cop1-4 hfr1. PP2A was used as an internal control. Wild type Col-0 was set as 1 . Error bars indicate standard deviation ( $\mathrm{n}=$ 3 independent biological repeats). (B) The PIL1 and XTR7 promoter occupancies of TAPPIF1 were reduced in cop1-4 background but increased in cop1-4hfr1 background. The ChIP-qPCR assays were performed on 3-day-old dark grown seeding expressing TAP-PIF1 fusion protein in cop1-4 and cop1-4hfr1 backgrounds. (C)Photographs of seedlings of wild type, cop1-4, cop1-4hfr1, cop1-4/TAP-PIF1 and cop1-4hfr1/TAP-PIF1. Seedlings were grown in the dark for 5 days. White bar $=5 \mathrm{~mm}$. (D) Bar graph showing hypocotyl lengths of various genotypes as described in (C). Error bars indicate standard deviation. Significant difference between different genotypes was determined using one-way ANOVA and Tukey's HSD tests, indicated by different letters. 


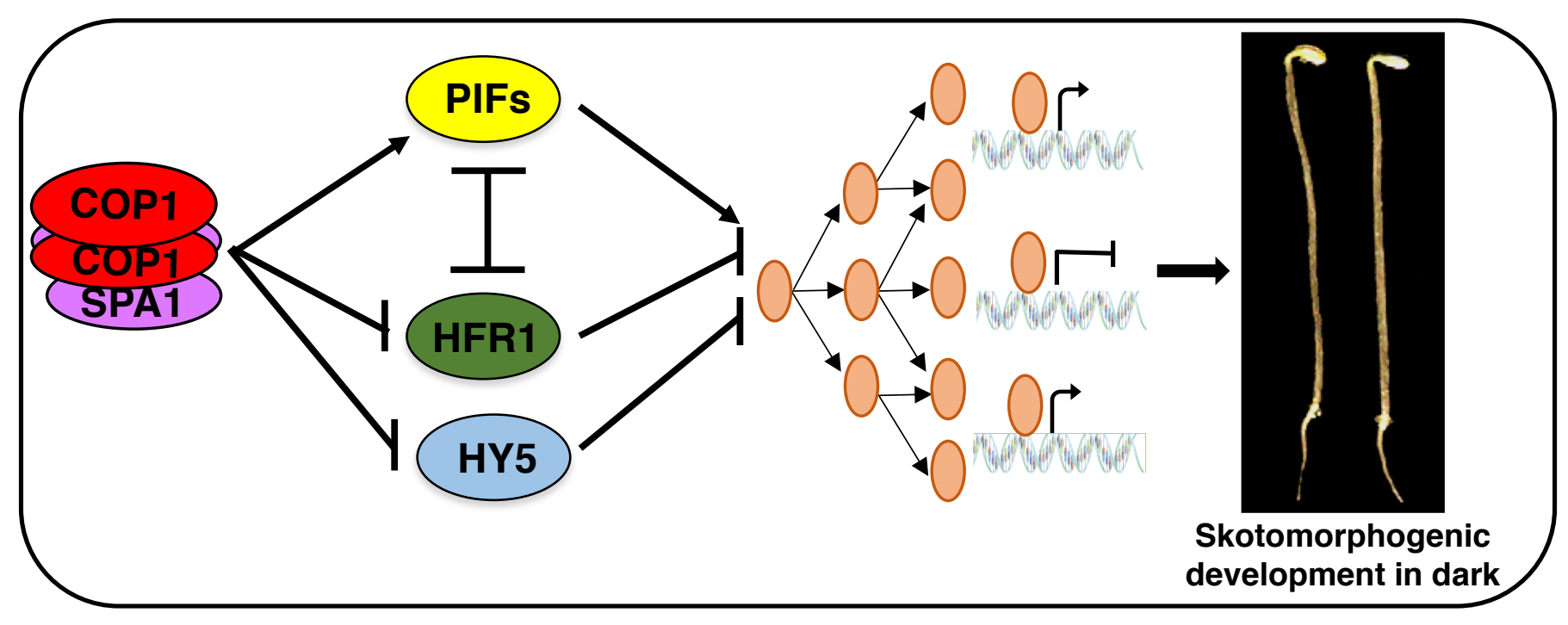

Figure 7: Model showing how COP1 and SPA proteins regulate various transcription factors to promote skotomorphogenesis in the dark.

Mutation in COP1, SPAs and PIFs results in constitutive photomophogenic (cop) phenotypes in the dark. PIFs and HFR1 reciprocally regulate their abundance, while HFR1 inhibits the PIF activity by sequestration. Regulation of these transcription factor abundance and activity by the COP1-SPA complex promotes skotomorphogenic development. 